\title{
MONITORING PERFORMANCE OF THE ADVANCED LIGHT SOURCE
}

\author{
W. Byrne, E. Lampo, B. Samuelson, LBNL, Berkeley, CA 94720 USA*
}

\section{Abstract}

Providing high quality light to users in a consistent and reliable manner is one of the main goals of the accelerator physics group at the Advanced Light Source (ALS). To meet this goal considerable time is spent monitoring the performance of the machine. At the Group's weekly meeting the performance of the accelerator over the previous week's run is reviewed. This paper describes the parameters that are monitored to optimize the performance of the ALS.

\section{INTRODUCTION}

The Advanced Light Source (ALS) is a $1.0-2.0 \mathrm{GeV}$ third generation synchrotron light source located at the Lawrence Berkeley National Laboratory. One of the main goals of the Accelerator Physics Group at the ALS is to provide high quality light to users in a consistent and reliable manner. To meet this goal, a large fraction of our time is spent monitoring the performance of the machine. At the weekly Operations Critique meeting, the Accelerator Physics Group and representatives from the Operations, Engineering and Controls Groups review the performance of the accelerator over the previous week's run. Presented here are graphs of various parameters that characterize the performance of the storage ring as discussed at these meetings.

Figures 1 and 2, along with Table 1, show the accelerator's performance for a typical week (December 5 $-11,2000)$. This was a $1.9 \mathrm{GeV}$ multibunch run. For this particular week the user beam began at 8:00 AM on the $5^{\text {th }}(1 / 3$ into day 1$)$ and ended at midnight on the $10^{\text {th }}$. The beginning of day 1 and all of day 7 were used for accelerator physics studies and accelerator maintenance.

\section{RELIABILITY}

A plot of beam current over time (Figure 1) is a measure of how reliably beam has been delivered. Beam dropouts are noted (Table 1) and the causes discussed by the group. Action is taken to correct recurring problems.

\footnotetext{
* Work supported by the Director, Office of Energy Research, Office of Basic Energy Sciences, Material Sciences Division, U.S Department of Energy, under Contract No. DE-AC03-76SF0098
}

December 5-11, 2000

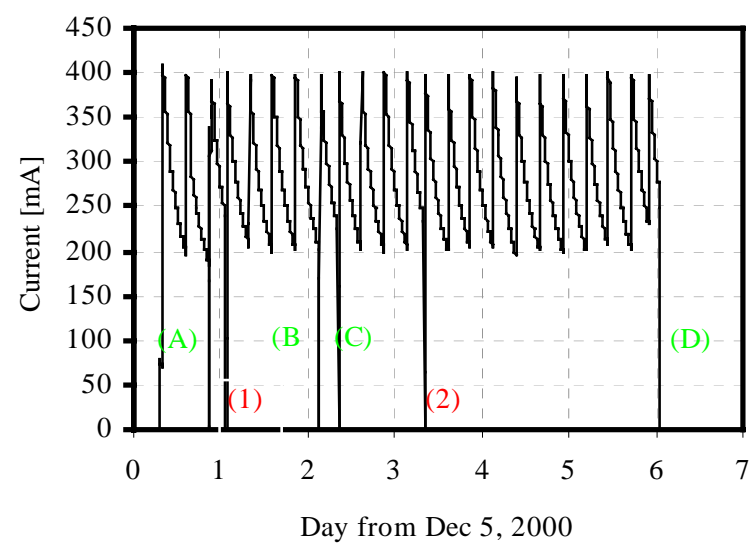

Figure 1: Beam current over time in the selected typical week, December 5-11.

Table 1: Causes of dropouts in the December 5-11 run.

\begin{tabular}{|c|c|c|c|c|}
\hline \multicolumn{5}{|c|}{ Drop Outs (Before Completion of Beam Delivery) } \\
\hline Outage & $\underline{\text { Date }}$ & $\underline{\text { Time }}$ & Description & Duration \\
\hline (1) & $12 / 6 / 2000$ & $1: 15$ & rf trip (Klystron Arc) & $25 \mathrm{~min}$ \\
\hline (2) & $12 / 8 / 2000$ & $8: 05$ & rf trip (Reverse Pwr) & $25 \mathrm{~min}$ \\
\hline \multicolumn{5}{|c|}{ Controlled Access, Early Refilling, or During Refill } \\
\hline Outage & Date & Time & Description & Duration \\
\hline (A) & $12 / 5 / 2000$ & 21:00 & Lost during refill & $30 \mathrm{~min}$ \\
\hline (B) & $12 / 7 / 2000$ & $3: 15$ & Change Camshaft buc & $3 \mathrm{~min}$ \\
\hline (C) & $12 / 7 / 2000$ & $8: 25$ & Change Camshaft buc & $3 \mathrm{~min}$ \\
\hline (D) & $12 / 11 / 2000$ & $1: 20$ & End of run & \\
\hline
\end{tabular}


Statistics are compiled each week to show how reliably fills were completed (Figure 2). For typical $1.9 \mathrm{GeV}$ operation, fills are scheduled every 6 hours. Each fill is categorized according to duration. At the end of the week the data is charted to show the percentage of fills that lasted the full 6 hours and the fraction that ended earlier than planned.

Our goal is to meet the scheduled 6-hour fill length $95 \%$ of the time. Presently, more than $90 \%$ of the fills meet this 6-hour goal.

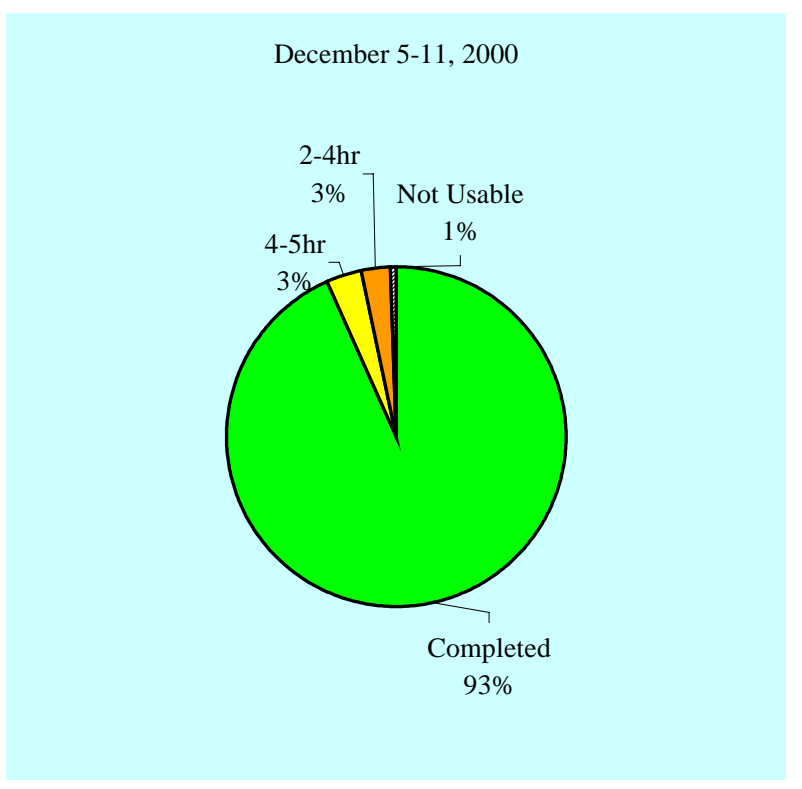

Figure 2: Fill statistics for the week of December 5-11, 2000. Percentage of fills that lasted the scheduled 6 hours, and percentage ending earlier than planned are shown.

\section{BRIGHTNESS, LIFETIME, CURRENT, AND BEAM SIZE}

The lifetime, $\tau$, in the ALS is predominantly determined by intrabeam (Touschek) scattering. As a result the lifetime is proportional to the transverse beam size, $\sigma_{\mathrm{x}}$ and $\sigma_{\mathrm{y}}$, and inversely proportional to current, $I$ :

$$
\tau \propto \frac{\sigma_{x} \sigma_{y}}{I}
$$

A figure of merit for brightness called the Touschek factor, Tau (Figure 3, fourth from top), is defined as

$$
T a u \propto \frac{I \tau}{\sigma_{x} \sigma_{y}}
$$

The brightness of the beam increases with Tau.
During a run, Tau should remain constant. Changes in Tau mean changes in the beam quality and are usually indicative of a problem with the machine. The parameters that determine the Touschek factor are plotted in Figure 3: beam current (top), beam lifetime (second from top), and beam size (third from top). Note: The apparently smaller beam size during most of the first day was a sensor artifact caused by a filter in the optical system that was too dense.

Almost all of the transverse size variation is due to the change in sensitivity of the measurement system with beam intensity. The real transverse variation is $1-2 \mu \mathrm{m}$.
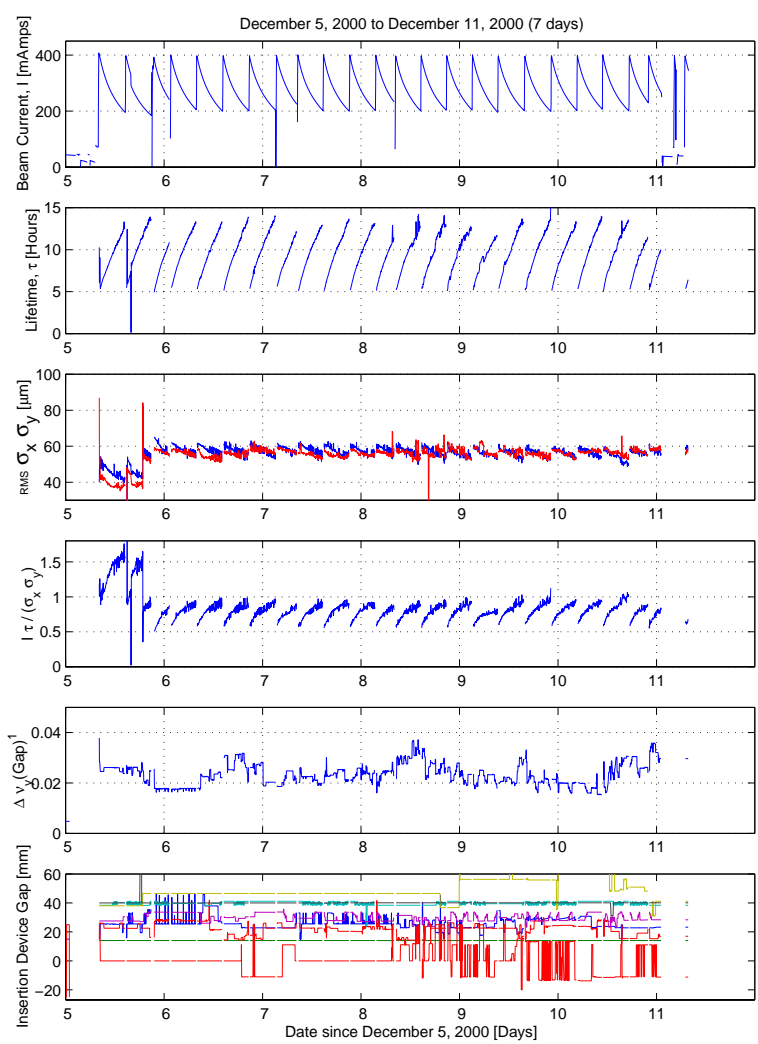

Figure 3: Beam parameters for a typical week (December 5-11, 2000). They include (top-bottom): beam current, beam lifetime, beam size, Touschek factor, tune changes and insertion device gap positions.

\section{POSITIONAL STABILITY}

In order to stabilize the beam position, the orbit of the beam in the storage ring is corrected once every second. This holds the beam position constant at the upstream and downstream ends of most of the straight sections. The performance of the correction algorithm is monitored routinely and adjusted if required to optimize performance. Figure 4 shows the vertical position of the beam at beam position monitors at the ends of the straight 
sections and in the curved section of Sector 9 over a week's run. The BPMs with large deviations in sectors 4, 6 and 9 were not in the correction loop. The position at the other BPMs, all of which were in the correction loop, remained constant within a few microns for the duration of the run.

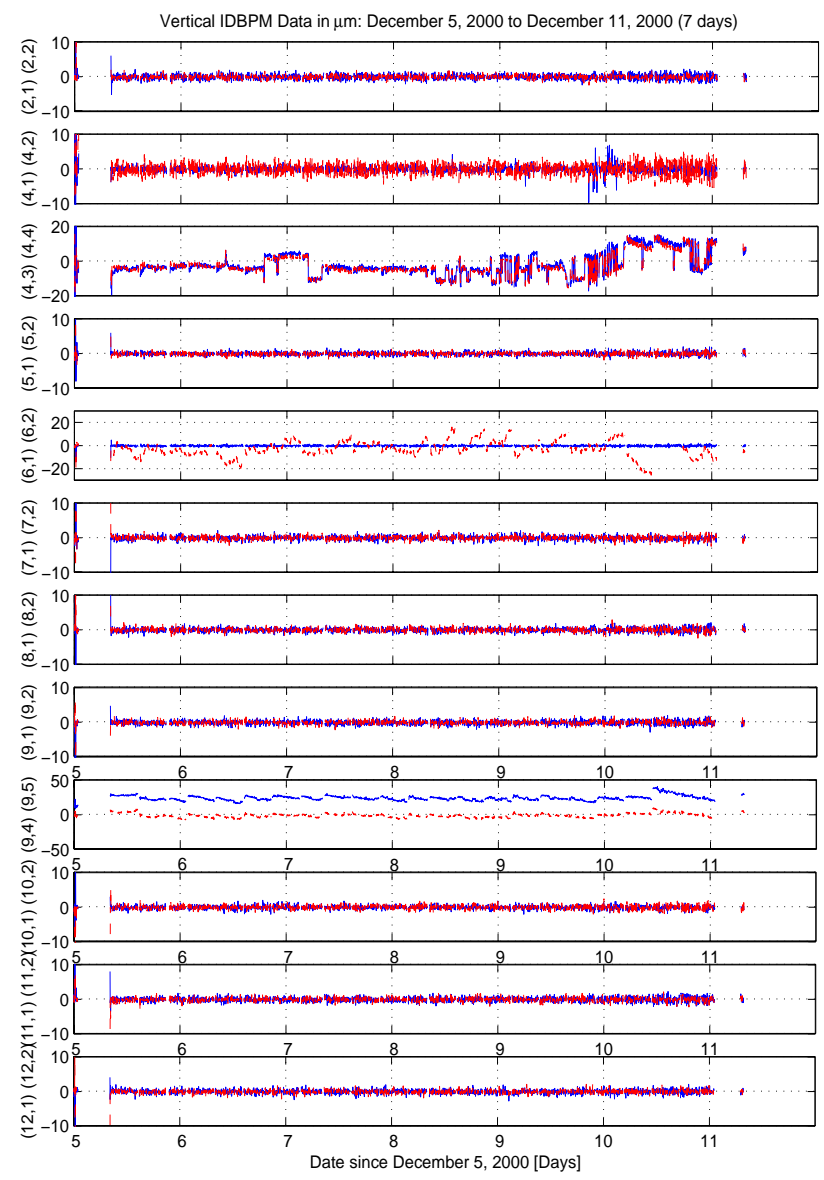

Figure 4: Vertical beam motion (December 5-11) at upstream and downstream ends of straight sections, in the center of sector 4 straight $(4,3 \& 4,4)$ and in the center of sector 9 arc $(9,4 \& 9,5)$.

\section{CONCLUSIONS}

The reliability and quality of the light provided for the users at the ALS improved substantially with the implementation of weekly machine performance reviews.

Beam delivery over time and reasons for beam dropouts were regularly monitored and systems causing lost operating time were identified. This allowed technical resources to be directed to the problem areas.

Review of the lifetime, beam size and Touschek factor plots provided insight into the performance of systems such as: the higher harmonic bunch lengthening cavities that enhance beam lifetime; the feedback systems that damp betatron and synchrotron oscillations; the feed forward algorithm that compensates tune shifts caused by insertion device gap changes.

Review of beam position stability data indicated how well the algorithm for orbit correction was working as well as the degree to which the feed forward routine compensated for dipole errors caused by insertion device gap changes. The stability of certain hardware, such as magnet power supplies, is also reflected in the beam stability plot.

In addition to reviewing these parameters at the weekly meeting, they are displayed in the Control Room in realtime. Most problems indicated by changes in these parameters can be corrected as they occur. 